\title{
Immune systems biology: immunoprofiling of cells and molecules
}

\author{
Aaron B. Kantor, Susan E. Alters, Karen Cheal, and Louis J. Dietz
}

BioTechniques 36:520-524 (March 2004)

White blood cells and their secreted products are key elements of immune systems biology that are important indicators of patient health and disease. We have developed the SurroScan ${ }^{T M}$ microvolume laser scanning cytometer to immunoprofile hundreds of variables, including cell populations, cell surface antigens, and intracellular molecules in antibody-based assays on small samples (about $1 \mathrm{~mL}$ ) of whole blood, processed blood, or other fluids without cell purification or washing steps. The system enables high-throughput, robust and automated data capture and analysis. We demonstrate the utility of this immunoprofiling technology platform by surveying patient samples before and after glucocorticosteroid administration and show both the expected and novel response characteristics. This system complements recent advances in genomic and proteomic approaches to disease prediction and monitoring.

\section{INTRODUCTION}

Various flow cytometry, image cytometry, and hematology instruments are available for analyzing cells $(1,2)$. Typically, a given platform is adapted for specific applications in a particular field, such as immunology, cancer biology, or microbiology. In the setting of the clinical laboratory, hematology analyzers are dedicated to the task of obtaining absolute cell counts of a few major blood cell types. Flow cytometers are deployed for immunophenotyping lymphomas and leukemias; they are also used for ongoing assessment of autoimmune and infectious diseases, and for monitoring transplantation. Image analyzers, including scanning cytometers, are used to characterize cell morphology, tissue histology, and nuclear DNA content of blood and solid neoplasms. They also play a role monitoring rare cancer cell events (3). In research and pharmaceutical laboratories, applications include high-throughput and high volume drug screening, highly polychromatic (more than six colors) analyses of immune system components in a few samples at a time (4), and high content analyses combining morphology, immunophenotyping, and biochemical measurements (5).

Despite the array of cytometry systems, there remains an unmet need for an instrument capable of broadly immunoprofiling large numbers of blood samples with the high-throughput and reproducibility suitable for use in clinical studies. The system should incorporate key attributes provided by other systems, including the ability to use whole blood and to obtain absolute cell counts, which are features common to hematology analyzers. It must perform immunophenotyping of multiple parameters, as is accomplished by flow cytometers, and it should provide the automation and throughput characteristic of screening ensembles. The cytometry platform should en- hance the understanding of systems biology and biomarker discovery in normal and pathophysiological conditions and in perturbations of the immune system caused by drugs. The SurroScan ${ }^{\mathrm{TM}}$ microvolume laser scanning cytometer (MLSC) together with reagents, disposable plastic capillary arrays, and software, allows rapid, sensitive, automated, and comprehensive cellular phenotyping for such applications.

\section{MATERIALS AND METHODS}

The SurroScan MLSC system (SurroMed, Menlo Park, CA, USA) derives in part from the Imagn2000 ${ }^{\mathrm{TM}}$ (BD Biosciences, San Jose, CA, USA) (6), but differs in that (i) four colors can be analyzed instead of two; (ii) capillary arrays are used to permit many more assays; and (iii) software streamlines data processing and connection to the database. Cellular assays were conducted on SurroScan using Flex32 ${ }^{\mathrm{TM}}$ capillary arrays (SurroMed) (7). Monoclonal antibodies and fluorescent tags were obtained from commercial vendors [BD Biosciences, BD Biosciences PharMingen (San Jose, CA, USA), Beckman Coulter (Fullerton, CA, USA), Serotec (Raleigh, NC, USA), and eBiosciences (San Diego, CA, USA)]. Three different fluorophores Cy ${ }^{\mathrm{TM}} 5$, Cy5.5 (8), and Cy7-allophycocyanin (Cy7-APC) $(9,10)$ were used as direct conjugates to the antibodies. Antibody-dye reagents were titrated to determine the appropriate concentration and combined into premade cocktails. They were then incorporated into a panel of 64 three-color homogeneous assays preformed on either whole or erythrocyte-lysed blood. Additional information on cellular assays is provided in Figure 1. Access to the SurroScan system is made available to SurroMed collaborators and clients. 
Sandwich-based enzyme-linked immunosorbent assay (ELISA) used antibody pairs obtained from commercial vendors and were detected with a chemiluminescent alkaline phosphatase substrate (CDP-Star ${ }^{\circledR}$; Applied Biosystems, Foster City, CA, USA) and read on the Wallac Victor plate reader (PerkinElmer Life and Analytical Sciences, Boston, MA, USA).

Eighty subjects were randomized and placed on a 3-day course of prednisone or placebo following informed consent to participate in the institutional review board approved protocol. Blood samples were collected in the morning following an overnight fast and processed within $6 \mathrm{~h}$ of collection. Samples from each subject were analyzed at two time points - before and after the treatment.

Drug and placebo groups were analyzed separately to identify significant differences between paired measurements before and after drug treatment using the paired Student's $t$ test or nonparametric test, as appropriate, for each variable.

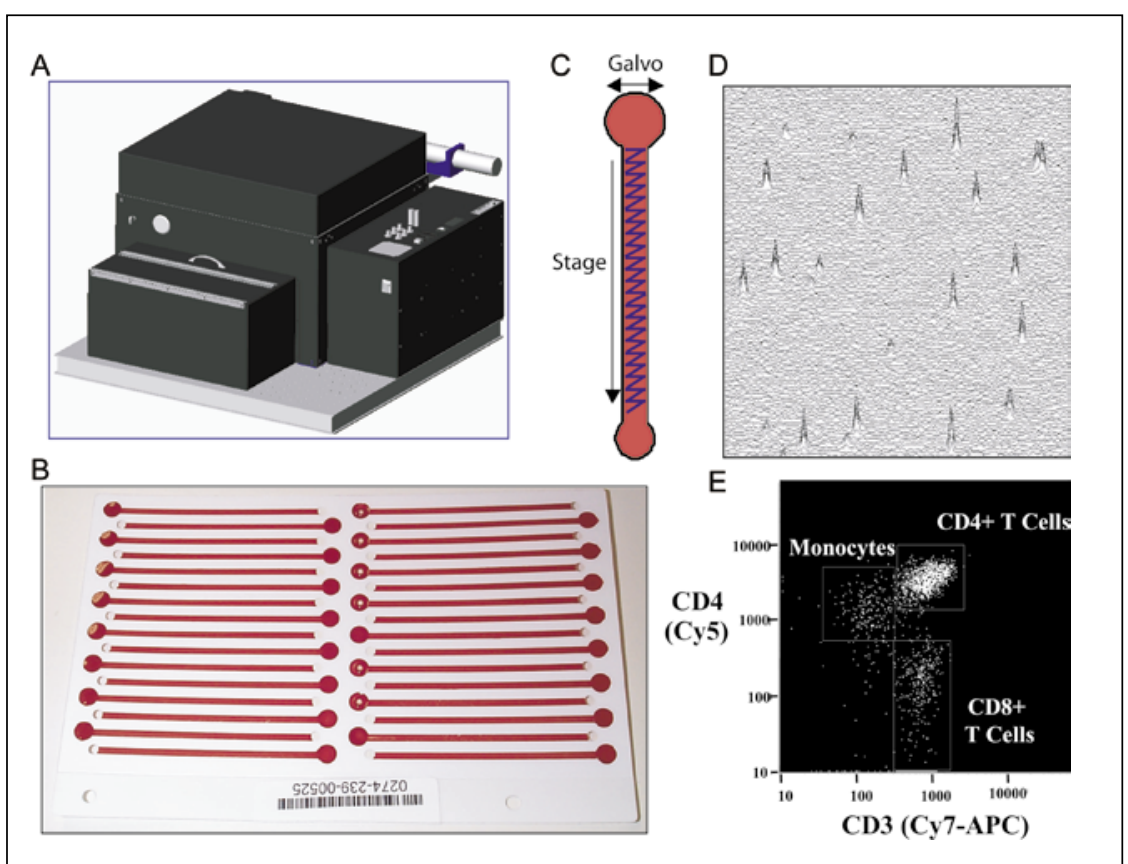

Figure 1. Cytometry system. (A) SurroScan instrument. The system uses confocal galvanometerbased raster scanning to image up to four fluorescent channels, each defined by a set of optical filters and measured with a separate photomultiplier tube. (B) Capillary array. Disposable capillary arrays are assembled from layers of polycarbonate sheets and adhesive. The array shown here holds 32 assays and has nine millimeter fill hole spacing for compatibility with multichannel pipets. Each capillary is 53 $\mathrm{mm}$ in length, $1.8 \mathrm{~mm}$ in width, and $135 \mu \mathrm{m}$ deep. The scan area is $1 \mathrm{~mm}$ wide and usually $40 \mathrm{~mm}$ long. The capillary is filled with whole blood that has been stained and appropriately diluted. (C) Scan pattern. A galvanometer moves the laser beam across the short dimension of an individual capillary while the stage moves in the long dimension. Successive capillaries are scanned in order. (D) Raster scan. Whole blood was labeled with Cy5-anti-CD4, Cy5.5-anti-CD8, and Cy7-allophycocyanin (APC) antiCD3 antibodies, diluted, and scanned. A raster scan generated for one fluorescence channel is shown. Peaks correspond to antibody-labeled cells identified with image processing software that produces a list-mode data file with parameters for every detected cell event (23). Unlabeled cells (erythrocytes and other leukocytes) are not identified. The background is principally from unreacted reagent that was not washed away. (E) Dot plot. Resultant data can be graphed with each dot representing one cell (customized version of FlowJo Cytometry Software; TreeStar, Ashland, OR, USA). Intensity data are compensated for spectral overlap, so the shown parameters are proportional to the amount of dye-antibody reagent on each cell. Standard gates, the boxes used to identify specific cell populations, are stored in a database for use throughout the study. Resultant information on cell counts and relative antigen levels on individual cell types are immediately available for study-wide analysis.
A total of 713 variables were analyzed together using SASTM Bonferroni $P$ value adjustment method of $\operatorname{Holm}(11,12)$ was used to maintain a study-wide $P$ value $<0.05$.

The SurroScan MLSC system (Figure 1) is used to classify and quantify hundreds of immune and inflammatory cell opulations and cellular antigens in small volumes of whole croscope slides, to present a mixture of cells and antibody reagents to the laser-based fluorescence imaging instrument analysis (Figure 1, A and B). In MLSC, as with flow cytometry, fluorophore-tagged antibody reagents specific ente enumerate specific leukocyte populations. In order to operate with whole blood and minimize the effects of autofluorescence and light attenuation, we use fluorophores that can be excited in the red and near infrared region $(>600 \mathrm{~nm})$ of the spectrum. Cy5, Cy5.5 (8), Cy7-APC $(9,10)$, and the Alexa Fluor ${ }^{\circledR}$ dyes $(13,14)$ work well in the system. The staining reaction can be done in whole blood or other single-cell suspensions. Peripheral blood mononuclear cells (PBMCs), erythrocyte-lysed blood, synovial fluid, bronchioalveolar lavage, splenocytes, and cell lines have been used in the system, as have viably frozen and thawed cells. In general, assays can be conducted in homogeneous mode. There is no need to wash the reagent away; quantitative dilution of the blood-antibody mixture is usually sufficient sample preparation. Addition of permeabilization and washing steps enable the monitoring of intracellular antigens. Each capillary array holds 32 separate assays and is compatible with multichannel pipeting devices. The cell-antibody mixtures are loaded into the capillaries and scanned.

In contrast to flow cytometry, the laser scans over stationary cells rather than cells flowing past the laser (Figure 1C). A small cylindrical laser beam is scanned through the capillary in one direction while the capillary is translated relative to the optical system in a second orthogonal direction. By scanning a predetermined volume for each capillary, absolute cell counts (cells $/ \mu \mathrm{L}$ ) are obtained directly. In this respect, the system is similar to hematology analyzers (2) and different from other laser scanning systems that focus on high-throughput screening (15-17), cell 
Table 1. Comparison of SurroScan and Flow Cytometry in Clinical Studies

\begin{tabular}{|lcc|}
\hline & SurroScan (MLSC) & Flow Cytometry \\
\hline Sample Preparation & & \\
Common & Whole blood & PBMC \\
Staining mode & No wash & $3 \times$ wash \\
Volume of blood needed $(\sim \mathrm{mL})$ & 1 & 10 \\
Time $(\mathrm{h})$ & 1 & $3-4$ \\
Assays/sample & 64 & 10 \\
Colors & $3-4$ & $3-6^{\mathrm{a}}$ \\
Scanning & & \\
Operation & Automated & Manual \\
Disposable & Flat capillary array & Tubes \\
Time (h, 64 assays) & 3 & 3 \\
Data Processing & Automated & Manual \\
Skilled analyst & Review & All steps \\
Absolute cell counts & Yes & Additional data needed \\
MLSC, microvolume laser scanning cytometer; PBMC, peripheral blood mononuclear cell. \\
aMore colors used in special research applications than clinical studies (4). \\
bCan be automated with extra equipment, but not common.
\end{tabular}

automated collection, processing, and analysis of the data. This data pipelining approach speeds data flow and reduces user error. The system is designed such that every cytometer yields the same result for a given sample. A combined instrument calibration and fluorescent tag compensation matrix is used to correct for spectral overlap among the fluorophores and to correct for optical differences among instruments. The compensation matrix is set at the beginning of a study using a combination of standard beads and stained blood samples and is monitored regularly. Adjustments are typically made monthly to accommodate instrument and reagent drift. Evaluation of CD4 and CD8 $\mathrm{T}$ cells on replicate blood samples on four different SurroScan instruments yielded a mean intra-instrument variation ranging from $3.3 \%$ to $5.4 \%(\mathrm{CV})$ for cell counts and $4.1 \%$ to $7.8 \%$ for intensities. Inter-instrument variation was slightly higher, ranging from $4.5 \%$ to $5.9 \%$ for cell counts and $8.0 \%$ to $10.4 \%$ for intensities. Consequently, common gates, the boxes used to identify specific

morphology (18), or rare cell (3) applications. An example of the resulting raster-scan profile for a single fluorescence channel is shown in Figure 1D. Each peak represents a single cell and can be converted to a list mode data file and displayed as a dot plot (Figure 1E). Typically, three antibody reagents, each with a different fluorescent tag and each detected in a different channel, are used per assay. The result is identifiable and quantifiable cell populations as indicated in the figure for CD4 T cells, CD8 T cells, and monocytes.

The SurroScan system is designed for the execution of many different assays on a large number of patient samples. Table 1 shows a comparison between the capabilities of this system versus flow cytometry. Here it can be seen that although the scanning times per individual assay are similar, the SurroScan system is much more efficient with respect to sample preparation and data analysis. Sample handling and operator interaction are minimized. There is no need to prepare PBMC by density gradients or to wash away unreacted reagent. The principal advantages of the system in terms of processing and absolute cell counts are for whole blood and, to a lesser extent, erythrocytelysed blood preparations. Informatics tools facilitate

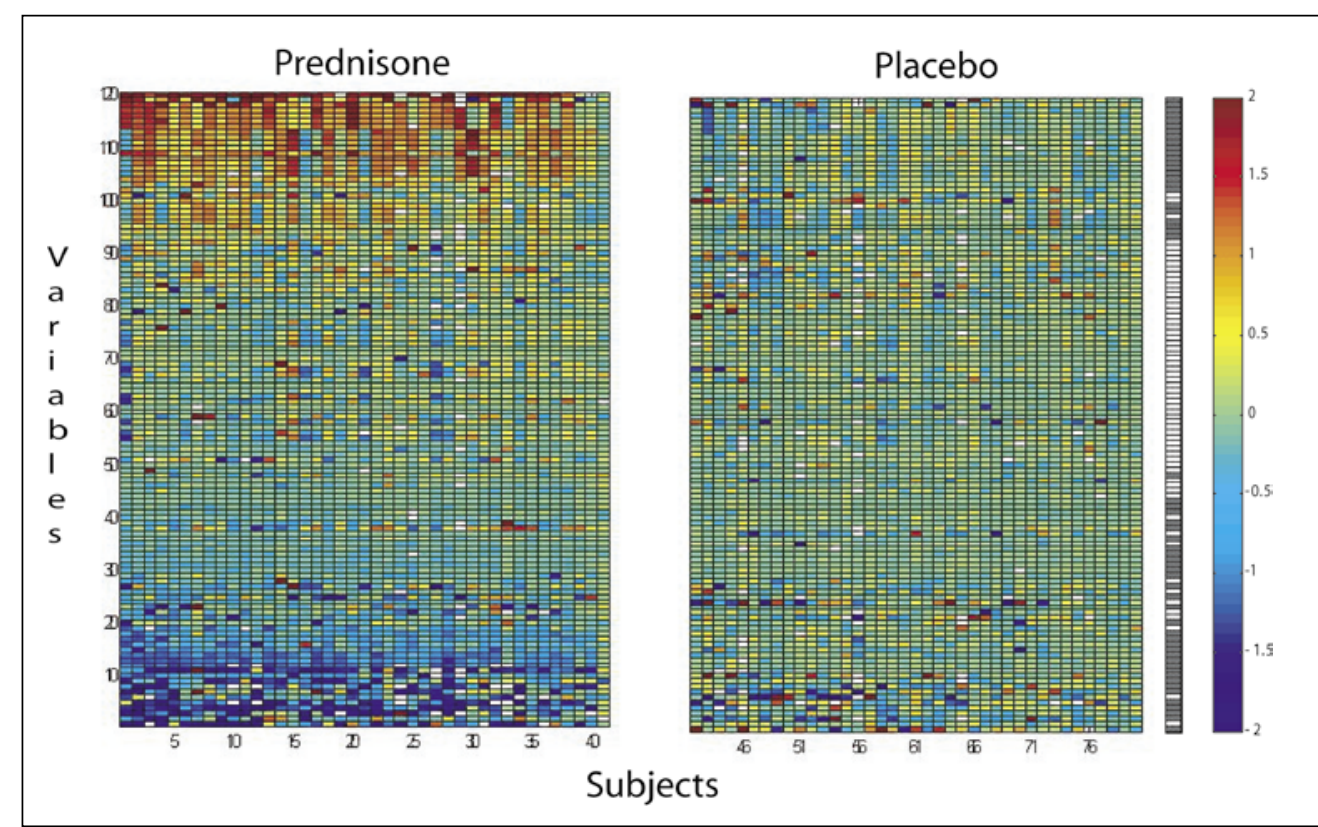

Figure 2. Pharmacodynamic profile. Data are shown for 120 representative variables on 80 subjects taking either prednisone $(n=41)$ or placebo $(n=39)$ for 3 days. The list of cell populations and corresponding variables can be found at http://www.BioTechniques.com/March2004/KantorSupplementary.htm]. Each pixel represents the $\log _{2}$ of the measurement taken after drug, divided by the measurement before drug. Color scale is shown on the right. Red represents an increase, and dark blue represents a decrease in the variable. Missing values are white. Significant changes were determined using the appropriate parametric or nonparametric paired test along with the step-down Bonferroni correction of Holm to protect against false positive errors and maintain a study-wide $P$ value of 0.05 . All analysis was done using SAS statistical software. Significant changes are indicated in gray in the column second from the right. Variables are sorted based on the largest changes observed for the subjects taking prednisone. 
We applied our immunophenotyping platform to the pharmacodynamic profiling of oral prednisone, a common glucocorticoid steroid. Glucocorticoids, both inhaled and systemic, have broad-spectrum anti-inflammatory and immunosuppressive effects and have long been used in the treatment of asthma and other diseases. However, they are associated with both subjective and objective side effects that limit their usefulness. A more comprehensive understanding of their pharmacodynamics is desirable. Eighty subjects were enrolled in a single-blind, placebo-controlled, randomized parallel study to evaluate the effects of prednisone on cellular and soluble bioanalytical parameters after 3 days of twice-a-day treatment with $20 \mathrm{mg}$ of prednisone or placebo per dose. Blood samples were analyzed before and after a 3-day treatment.

In this study, a panel of 64 three-color cellular assays and 67 immunoassays for soluble proteins was used. The cellular assays are arranged in two disposable capillary arrays and allow the identification and enumeration of hundreds of different cell types and cell-associated molecules that are relevant to immune, inflammatory, and metabolic processes. Each reagent cocktail typically contains one or two antibodies to the major cell populations-neutrophils, eosinophils, monocytes, T cells, B cells, and natural killer (NK) cells, plus one or two antibodies to subset antigens, which may indicate the functional state, activation state, or adhesion characteristics of the major cell population. Soluble proteins are measured with sandwich ELISA using matched antibody pairs and chemiluminescent detection. Analytes include immunoglobulin isotypes, cytokines and cytokine receptors, chemokines, acute phase proteins, soluble adhesion molecules, matrix metalloproteinases (MMPs), and their inhibitors.

We observed many significant changes post-prednisone treatment, but not post-placebo treatment. Paired Student's $t$-tests and nonparametric tests were used to evaluate the changes as appropriate for each variable. A conservative statistical procedure, the step-down Bonferroni method of Holm, was used to protect against false positive errors and to calculate an adjusted $P$ value for all comparisons. Paired tests yielded 199 significant changes with prednisone, but only one for placebo, allowing a high degree of confidence in the results. Differences were observed using all aspects of the bioanalytical platform, cell counts, cell surface antigen levels, and soluble factors.

A distinct pattern of changes is observed with the prednisone group. Figure 2 shows a representative data set, including 120 of the 713 variables measured. Variables are sorted based on the degree of change observed with the drug, and significant changes are indicted with gray shading in the sidebar. Significant changes are observed in cell populations. For example, circulating neutrophils, neutrophil subsets, B cells, and mono- 
cytes are increased, whereas circulating eosinophils and CD4 $\mathrm{T}$ cell subsets are decreased. There are also significant changes in levels of cell surface expression. For example, human leukocyte antigen (HLA) class II is decreased on both B cells and monocytes. Significant changes are also observed in soluble factors, with MMP-3 (stromelysin) increased by 5 -fold.

In summary, the SurroScan system is a breakthrough technology platform that enables immunoprofiling of small quantities of whole blood for drug response and disease indicators in a format that can analyze hundreds of parameters at one time. This expands by more than an order of magnitude methods currently in routine use and could have a major impact of patient diagnosis and management. The SurroScan technology is well suited to be part of an integrated bioanalytical platform for clinical research and to facilitate comprehensive differential phenotyping of patient samples for the discovery of biomarkers. This platform is complementary to and can be easily combined with other bioanalytical technologies such as proteomic and metabolomic profiling with mass spectrometry (19), gene expression arrays (20), and protein expression arrays $(21,22)$.

\section{ACKNOWLEDGMENTS}

We thank Harini Govindarajan, Brent Reynolds, and Chad Minks for processing clinical samples and completing cellular assays; Brad Brown, Natalia Tsekhonovskaya, and Remy Cromer for completing immunoassays; Chris Todd, Alex Puski, and Ian Walton for setting-up and maintaining SurroScan instruments; Pierre Hyun and Naomi Freiman for informatics support; and Andrea Perrone for clinical operations. We thank Dr. Alan Heller, Christy Schroeder, Dr. Theodore Chu, Kristin Chelslew, and Dr. Harold Guy for providing subject samples for the study. We thank Michael Derks, Randy Batenhorst, and Jim Snapper for contributions to the clinical protocol. We thank Mark Davis and Howard Schulman for thoughtful comments on the manuscript and Ron Krietemeyer for assistance with the figures. This work is supported, in part, by National Institute of Standards and Technology Advanced Technology Program (NIST ATP) award no. 7ONANBOH3OOO.

\section{REFERENCES}

1.Shapiro, H.M. 2003. Practical Flow Cytometry. John Wiley and Sons, Hoboken, NJ.

2.Groner, W. and E. Simson. 1995. Practical Guide to Modern Hematology Analyzers. John Wiley and Sons, Chichester, England.

3.Tibbe, A.G., B.G. de Grooth, J. Greve, P.A. Liberti, G.J. Dolan, and L.W. Terstappen. 1999. Optical tracking and detection of immunomagnetically selected and aligned cells. Nat. Biotechnol. 17:1210-1213.

4.De Rosa, S.C., J.M. Brenchley, and M. Roederer. 2003. Beyond six colors: a new era in flow cytometry. Nat. Med. 9:112-117.

5.Darzynkiewicz, Z., E. Bedner, X. Li, W. Gorczyca, and M.R. Melamed. 1999. Laser-scanning cytometry: a new instrumentation with many applications. Exp. Cell Res. 249:1-12.

6.Dietz, L.J., R.S. Dubrow, B.S. Manian, and N.L. Sizto. 1996. Volumetric capillary cytometry: a new method for absolute cell enumeration. Cytometry 23:177-186.

7.Walton, I.D., L.J. Dietz, G. Frenzel, J. Chen, J. Winkler, S. Norton, and
A.B. Kantor. 2000. Microvolume laser scanning cytometry platform for biological marker discovery, p. 192-201. In Proc.SPIE-Int.Soc.Opt.Eng. IBOS Society of Photo-Optical Instrumentation Engineers.

8.Mujumdar, R.B., L.A. Ernst, S.R. Mujumdar, C.J. Lewis, and A.S. Waggoner. 1993. Cyanine dye labeling reagents: sulfoindocyanine succinimidyl esters. Bioconjug. Chem. 4:105-111

9.Roederer, M., A.B. Kantor, D.R. Parks, and L.A. Herzenberg. 1996. Cy7PE and Cy7APC: bright new probes for immunofluorescence. $\mathrm{Cy}$ tometry 24:191-197.

10.Beavis, A.J. and K.J. Pennline. 1996. Allo-7: a new fluorescent tandem dye for use in flow cytometry. Cytometry 24:390-395.

11.Holm, S. 1979. A simple sequentially rejective multiple test procedure Scand. J. Stat. 6:65-70.

12.Blair, R.C., J.F. Troendle, and R.W. Beck. 1996. Control of familywise errors in multiple endpoint assessments via stepwise permutation tests. Stat. Med. 15:1107-1121.

13.Panchuk-Voloshina, N., R.P. Haugland, J. Bishop-Stewart, M.K. Bhalgat, P.J. Millard, F. Mao, and W.Y. Leung. 1999. Alexa dyes, a series of new fluorescent dyes that yield exceptionally bright, photostable conjugates. J. Histochem. Cytochem. 47:1179-1188.

14.Berlier, J.E., A. Rothe, G. Buller, J. Bradford, D.R. Gray, B.J. Filanoski, W.G. Telford, S. Yue, et al. 2003. Quantitative comparison of long-wavelength Alexa Fluor dyes to Cy dyes: fluorescence of the dyes and their bioconjugates. J. Histochem. Cytochem. 51:1699-1712.

15.Zuck, P., Z. Lao, S. Skwish, J.F. Glickman, K. Yang, J. Burbaum, and J. Inglese. 1999. Ligand-receptor binding measured by laser-scanning imaging. Proc. Natl. Acad. Sci. USA 96:11122-11127.

16.Martens, C., A. Bakker, A. Rodriguez, R.B. Mortensen, and R.W. Barrett. 1999. A generic particle-based nonradioactive homogeneous multiplex method for high-throughput screening using microvolume fluorimetry. Anal. Biochem. 273:20-31.

17.Swartzman, E.E., S.J. Miraglia, J. Mellentin-Michelotti, L. Evangelista, and P.M. Yuan. 1999. A homogeneous and multiplexed immunoassay for high-throughput screening using fluorometric microvolume assay technology. Anal. Biochem. 271:143-151.

18.Kamentsky, L.A. 2001. Laser scanning cytometry. Methods Cell Biol 63:51-87.

19.Aebersold, R. and M. Mann. 2003. Mass spectrometry-based proteomics. Nature 422:198-207.

20.Chee, M., R. Yang, E. Hubbell, A. Berno, X.C. Huang, D. Stern, J. Winkler, D.J. Lockhart, et al. 1996. Accessing genetic information with high-density DNA arrays. Science 274:610-614.

21.Robinson, W.H., C. DiGennaro, W. Hueber, B.B. Haab, M. Kamachi, E.J. Dean, S. Fournel, D. Fong, et al. 2002. Autoantigen microarrays for multiplex characterization of autoantibody responses. Nat. Med. 8: 295-301.

22.MacBeath, G. and S.L. Schreiber. 2000. Printing proteins as microarrays for high-throughput function determination. Science 289:17601763.

23.Norton, S.M., J. Winkler, and L.J. Dietz. 2000. Cell enumeration and characterization in microvolume laser scanning cytometry: a multicolor image processing package, p. 20-30. In Proc.SPIE-Int.Soc.Opt.Eng. IBOS Society of Photo-Optical Instrumentation Engineers.

Address correspondence to Aaron B. Kantor, SurroMed, Inc., 1430 O'Brien Drive, Menlo Park, CA 94025, USA. e-mail: akantor@surromed.com 